\title{
OPTIMIZATION OF INLET VALVE LEAFLET SHAPE USING METAMODEL AND FLUID-STRUCTURE INTERACTION
}

\section{LAURA PALYS ${ }^{1}$, MIROSŁAW W. MRZYGLÓD ${ }^{2}$, MACIEJ GAWLIKOWSKI ${ }^{3}$, ROMAN MAJOR ${ }^{4}$}

\author{
${ }^{1}$ Opole University of Technology \\ Mikołajczyka 5, 45-271 Opole, Poland \\ 1'laura.palys@doktorant.po.edu.pl \\ ${ }^{2}$ Opole University of Technology \\ Mikołajczyka 5, 45-271 Opole \\ m.mrzyglod@po.edu.pl \\ ${ }^{3}$ Silesian University of Technology \\ Roosevelta 40, 41-800 Zabrze, Poland \\ maciej.gawlikowski@polsl.pl \\ ${ }^{4}$ Institute of Metallurgy and Material Science \\ Reymonta 25, 30-059 Kraków, Poland \\ r.major@imim.pl
}

Key words: Shape optimization, Metamodeling, Fluid-structure interaction, Ventricular assist device, Leaflet valve

\begin{abstract}
Cardiac assist devices like continuous flow ventricular assist devices (CF-VAD) provide several benefits, including improved durability or higher energy efficiency. This paper presents the shape optimization for the inlet valve geometry of the pulsatile VAD using a metamodeling framework and fluid-structure interaction (FSI). The main task of the inlet valve is preventing the backflow to occur and keep proper valve washing as well as low hemolysis, induced by high shear stress. The finite element (FE) model of the valve was generated using a parametric model. The FE model was associated with a fluid flow analysis environment. The shear stress at wall leaflet structures was observed for all design points. For selected design variables, the minimization of the leaflet's wall shear stresses was carried out. As a result of optimization, the optimal valve leaflet shape was found. The developed modeling methodology can be easily adapted to investigate biomedical problems especially in the process of creating devices supporting cardiac circulation.
\end{abstract}

\section{INTRODUCTION}

Until the 1960s, the era of continuous improvement of cardiac assist devices began. The advent of the first, implantable artificial ventricle device was reported in 1963 [1] and it is considered as the beginning of a modern approach for the development of ventricular assist device (VAD). The first devices to support the work of the heart were pneumatically driven and were designed to recreate the native cardiac cycle. All initial constructions were characterized by large dimensions, weights, and an external drive, which limited patient mobility. The next 
stage in the improvement of the cardiac assist device is the development of devices with continuous flow pumps [2]. Parameters considered in the construction of cardiac assist device are blood damage: hemolysis and thrombosis induced by non-physiological flow conditions such as recirculation areas, sharp edges, narrow clearances but also fields with low shear stresses, velocities causing stagnation areas. In the following article, the authors try to explore the topic of PVAD's element optimization, and thus make it safer for the patient. The topic is extremely important because it is related to human health and life, and in the future, it may be developed into issues related to the extension of the PVAD's working time and the biocompatibility of the materials used in its design. The biggest driving force in the search for cardiac assist device solutions was the discovery of the possibilities of the computational fluid dynamics (CFD) software. To make good use of CFD methods, the obtained solution was implemented to develop structural domains, especially to predict the deformation of objects and acting forces. From these two areas, a new type of analysis has emerged, which we call fluidstructure interaction (FSI). Two-way analyses are of particular importance here, in which both fluid and material deformation influence each other. The general concept of CFD includes various methods of solving flow problems. Most of the articles focus on understanding the structure of devices using commercial software, which is most often based on solving NavierStokes equations using mesh methods and the Reynolds-averaged Navier-Stokes (RANS) approach for turbulence modeling. The increase in computing power influenced the performance of more complex and comprehensive numerical analyses on cardiac assist devices. The vast majority of investigations base their scheme on non-systematized measurement points One example of such an approach is work [3]. To solve the problem, the meshless method,
(Smoothed Particle Hydrodynamics) was used, three variables of the impeller blade pitch angles
and two gap sizes between the rotor and housing were analyzed in the continuous flow VAD.
Another work [4] presented an optimization with three motion profiles of pusher plate, three-
stroke, three pulsatile rates, and two assist modes were implemented to evaluate blobd damage. Calculations were made with FSI analysis based on the mesh method and turbulence modeling

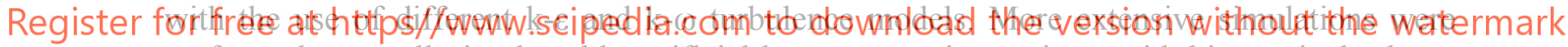
performed on totally implantable artificial heart supporting patients with bi-ventricular heart failure [5]. In addition to the operation of a mechanical device, the effects on the aortic and mitral valves were examined. Consequently, differences in the distribution of velocities have a direct impact on the shear stress, the relationship of which in the blood damage indexes has been repeatedly confirmed [6]. There is a small number of works in the literature dealing with the optimization of heart support devices. These studies are limited due to the large area of search, among which it is necessary to perform complex and computationally demanding analyses. One example of a planned optimization of such a device is work evaluating various impeller and volute shapes [7]. The techniques that were used in process of optimization: sampling - Sobol sequences with eleven variables, metamodel: Artificial neural network, optimal points: Non-dominated Sorting Genetic Algorithm-II. The whole process led to the improvement of the hemolysis index and pump hydraulic efficiency. However, it is worth noting that the research used some simplifications like steady flow and Newtonian's blood model assumption. In the next work [8], the surrogate management framework, a derivativefree pattern search optimization method was used. For objective function, the particle residence time is selected, which is directly related to thrombotic risk. Four variables in the construction of the inlet and outlet arms were investigated to evaluate optimal pulsatile ventricular assist 
device (PVAD) geometry. The authors conclude that the vertical orientation of the channels results in the uniformity of the flow from inflow to outflow, decreasing fluid residence time. Overall, these studies highlight the need for taking into account many variables, because some simplifications cause deviations from the actual results. Developing one universal model requires a lot of work, but considering single problems allows leaving behind the guesswork elements, that do not have a significant impact on the research. All of the optimization studies reviewed here support the hypothesis that PVAD is a suitable, interesting device for which the developed optimization methods allow to limit the number of computer simulations, at the same time ensure for very accurate results.

The article will present the optimization of the shape for the geometry of the VAD pulse inlet valve. To find the optimal geometrical parameters of the structure, the methodology of metamodeling and the coupling with the fluid-structure interaction simulation (FSI) environment will be used.

\section{DEVELOPMENT OF A VALVE METAMODEL}

The first step in this process was to create the geometry of the valve inlet. The structure of the leaflet valve was formed by the intersection of the inflow cannula with the blood chamber. The resulting plane was pulled in both directions to a thickness of $0.3 \mathrm{~mm}$. The geometry of the generated part (blood chamber, inflow cannula, valve) is shown in Bląd! Nie można odnaleźć źródla odwolania. The structure of the leaflet was strengthened with $0.1 \mathrm{~mm}$ thick metal mesh.
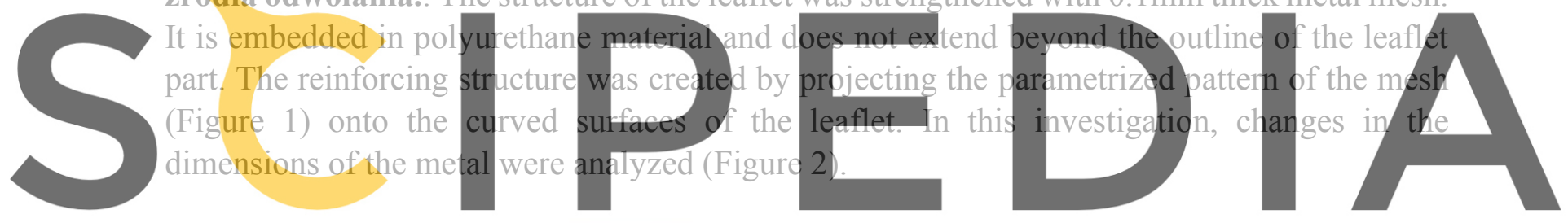

Register for free at https//www.scipedia.com to download the version without the watermark

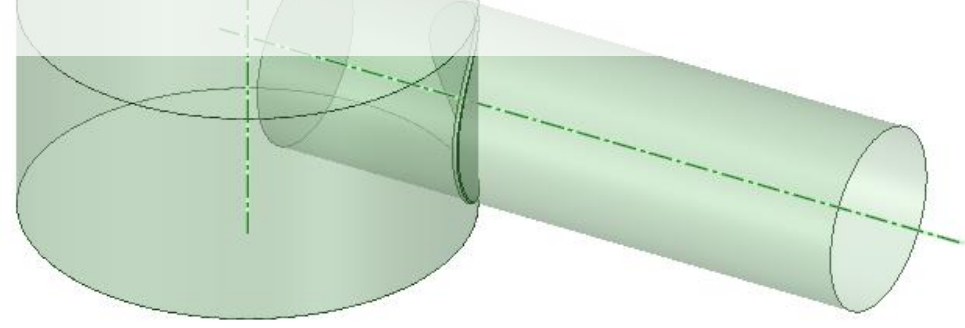

Figure 1.Surfaces used in the process of generating the valve geometry.

The most desired features in the material used in biomechanical engineer are biocompatibility, hemocompatibility, wide mechanical properties. Also, in this case, its benefits were used and numerical simulations were carried out using polyurethane as leaflet material. The properties of stainless steel were assumed for the reinforcing mesh. Properties of the materials used in the construction of the valve are presented in Table 1. 
a)

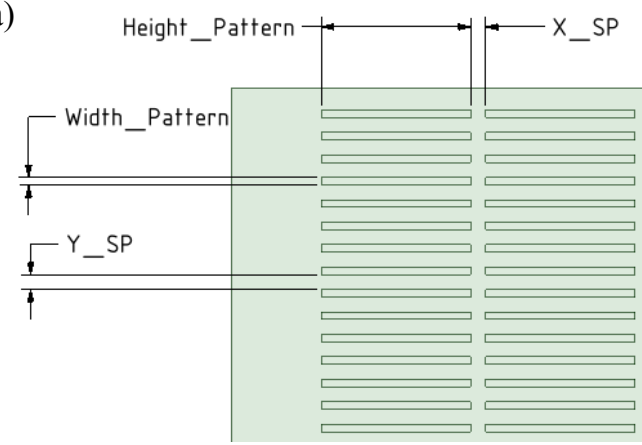

b)

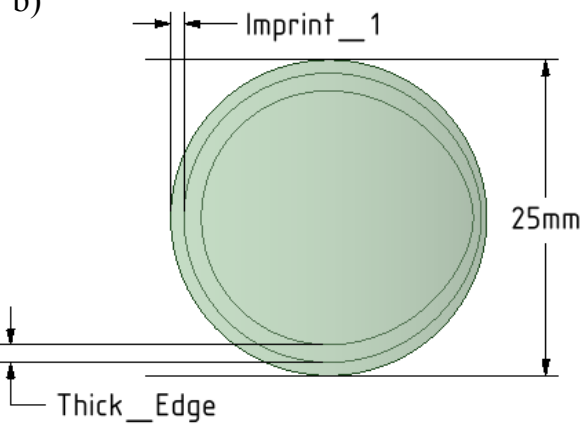

Figure 2. a) Stiffener mesh patter and b) metal geometry contour limiter

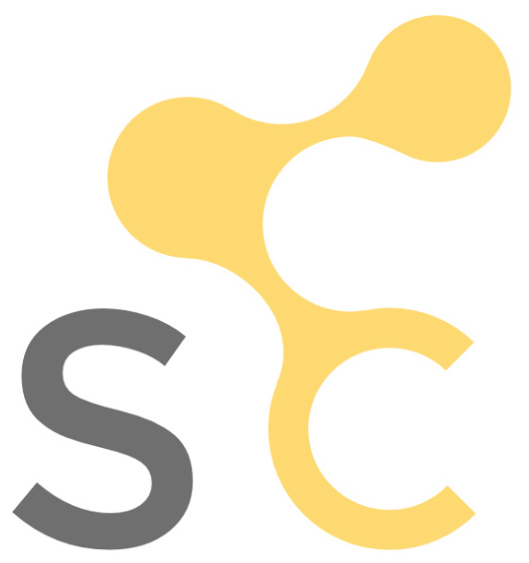

Table 1. Material properties of inflow valve.

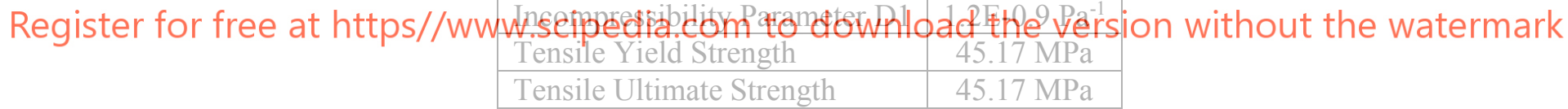

In the numerical procedure, blood is approximated as Newtonian or Non-Newtonian fluid, where many submodels are developed. Their compliance with the experimental results can be found in works [9], [10]. It was generally assumed that the difference between the Newtonian and the non-Newtonian models relay in the dissimilarity during the flow in microstructures, recirculation, separation region, or low Reynolds number operation of low. In this case, the Carreau model (Eq.1) was used and its parameters [9] can be found in Table 2.

The numerical simulation was performed using commercial software Ansys (ANSYS, Inc.). Two modules for numerical analysis were used to solve this problem; Transient Structural and Fluent, both of these components were coupled using System Coupling. Data exchange between modules can be transferred: from fluid to structure domain, with no response to changes in structure (One-Way FSI); from fluid to the structure domain, but structure changes and the information returns to the fluid and influences the nature of the flow (Two-Way FSI). Taking 
into account the dimensions, material and large deformations in the valve, it was necessary to apply two-way FSI analysis. The cross-section of the valve and the fluid domain is shown in

$$
\eta=\eta_{\infty}+\left(\eta_{0}-\eta_{\infty}\right) \cdot\left[1+(\lambda \gamma)^{2}\right]^{\frac{n-1}{2}}
$$

Table 2.Blood properties.

\begin{tabular}{|cl|c|}
\hline \multicolumn{3}{|c|}{ Blood properties } \\
\hline$\rho$ & blood density & $1050 \mathrm{~kg} / \mathrm{m}^{3}[11]$ \\
\hline$\eta$ & non-Newtonian viscosity & \\
\hline$\eta_{0}$ & zero-shear-rate viscosity & $0.056 \mathrm{~Pa} \cdot \mathrm{s}$ \\
\hline$\eta_{\infty}$ & infinite-shear-rate viscosity & $0.00345 \mathrm{~Pa} \cdot \mathrm{s}$ \\
\hline$\lambda$ & characteristic time & $3.313 \mathrm{~s}$ \\
\hline $\mathrm{n}$ & nower-law index & 0.3568 \\
\hline$\gamma$ & shear rate & \\
\hline
\end{tabular}
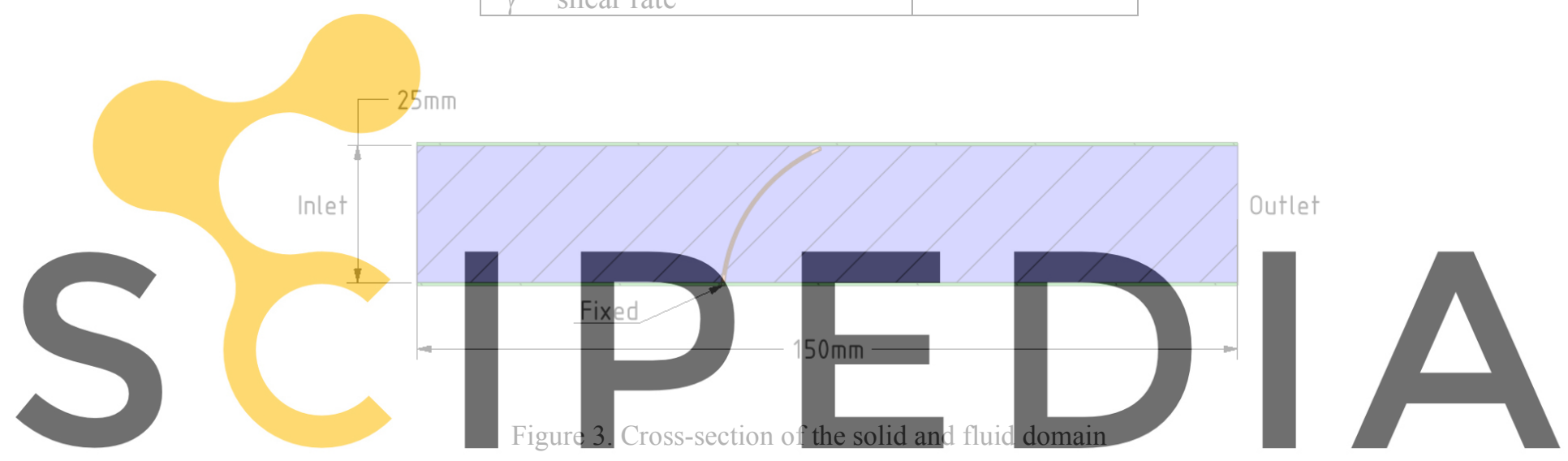

Register for free at https//www.scipedia.com to download the version without the watermark

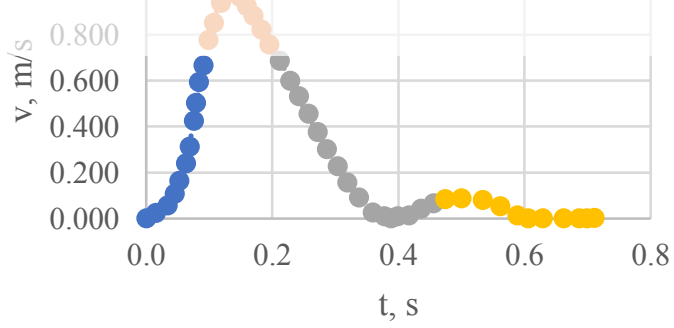

Figure 4. Time-varying velocity profile at the inlet of the canal.

The leaflet and the reinforcing structure were meshed with hexahedral and tetrahedral elements, with a minimum number of three on the element thickness. The minimum size of the elements was set to $0.0002 \mathrm{~mm}$. One end of the valve has been fixed. The area of data exchange with the fluid domain was marked on the surface of the leaflet. The fluid domain consisted entirely of tetrahedral elements. The k-omega SST turbulence model was selected as the most frequently used in the simulation of VADs and blood flow, because of satisfactory agreement 
between simulation and experiment data [12]. Substantial deformations in the valve structure required the use of a dynamic mesh in which smoothing and re-meshing options were enabled. For velocity-inlet, time-dependent values were assumed, which are presented in Figure. All the results showed in further consideration are presented for velocity-inlet equals $0.11 \mathrm{~m} / \mathrm{s}$.

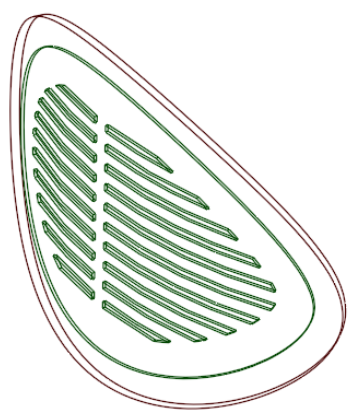

Figure 5. Inlet valve structure.

In this analysis, the number of measurement points reached 10. The parameterized valve geometry is shown in Figure 5, where the purple outline presents the leaflet and green metal stiffener.
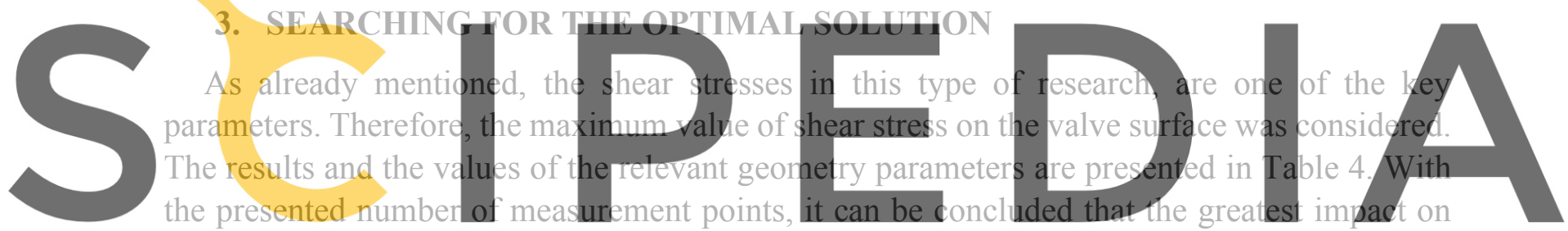

the formation of the maximum shear stress level results from the arrangement of the reinforcing

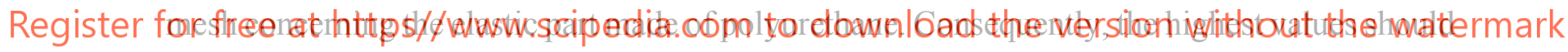
occur precisely in those areas.

Table 3.Input parameter values and results.

\begin{tabular}{|c|c|c|c|c|c|}
\hline DP & $\begin{array}{c}\text { Imprint_1, } \\
\mathrm{mm}\end{array}$ & $\begin{array}{c}\text { Thick_Edge, } \\
\mathrm{mm}\end{array}$ & $\begin{array}{c}\text { Height_Pattern, } \\
\mathrm{mm}\end{array}$ & $\begin{array}{c}\text { Width_Pattern, } \\
\mathrm{mm}\end{array}$ & $\begin{array}{c}\text { MaxWallShear, } \\
\text { Pa }\end{array}$ \\
\hline 1 & -1.05 & -0.35 & 5.25 & 5.25 & 21.65 \\
\hline 2 & -2 & -0.35 & 5.25 & 5.25 & 12.84 \\
\hline 3 & -0.1 & -0.2 & 10 & 0.5 & 46.23 \\
\hline 4 & -2 & -0.5 & 0.5 & 10 & 19.68 \\
\hline 5 & -0.1 & -0.5 & 0.5 & 10 & 21.13 \\
\hline 6 & -2 & -0.2 & 0.5 & 10 & 18.14 \\
\hline 7 & -0.1 & -0.2 & 0.5 & 10 & 33.00 \\
\hline 8 & -2 & -0.5 & 10 & 10 & 19.01 \\
\hline 9 & -0.1 & -0.5 & 10 & 10 & 33.11 \\
\hline 10 & -0.1 & -0.2 & 10 & 10 & 32.44 \\
\hline
\end{tabular}


Confirmation in the preliminary numerical tests can be observed on the contours of the shear stress distributions for individual measuring points ( see Figure ). At least in the initial phase, the greatest shear stresses (46 Pa) occur on the margin of the valve and their value decreases with increasing the distance between the mesh and the edges of the gate (up to $12.8 \mathrm{~Pa}$ ). As already mentioned, shear stress can affect various blood contents. Due to their different properties, maximum stress, and exposure time, blood platelets are less resistant to their degradative effects, than red blood cells. The photo also shows the shape of the stiffener on which the given shear stress is distributed.

a)

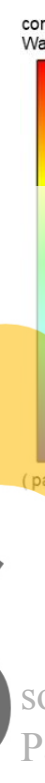

b)

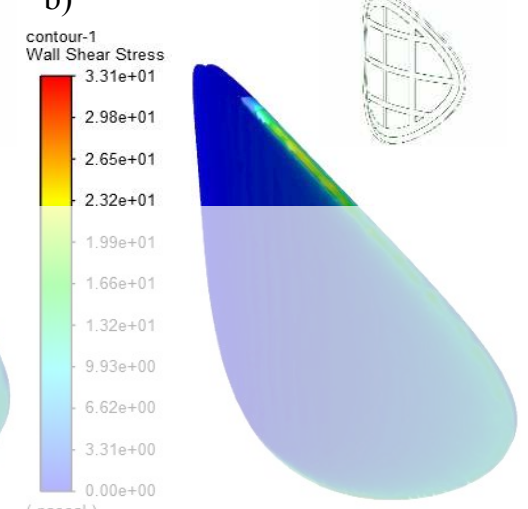

c)

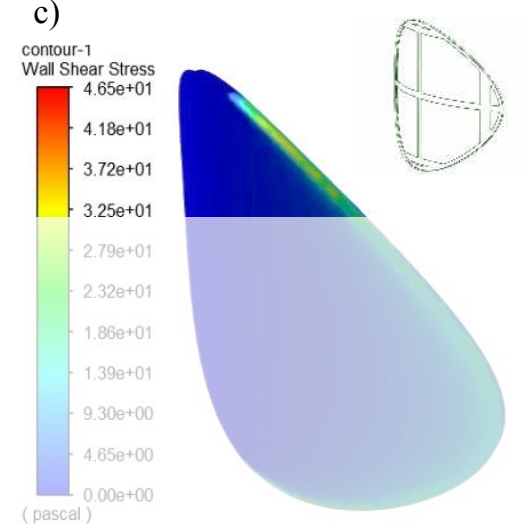

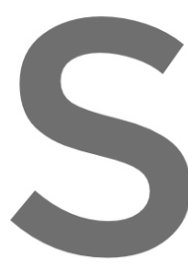
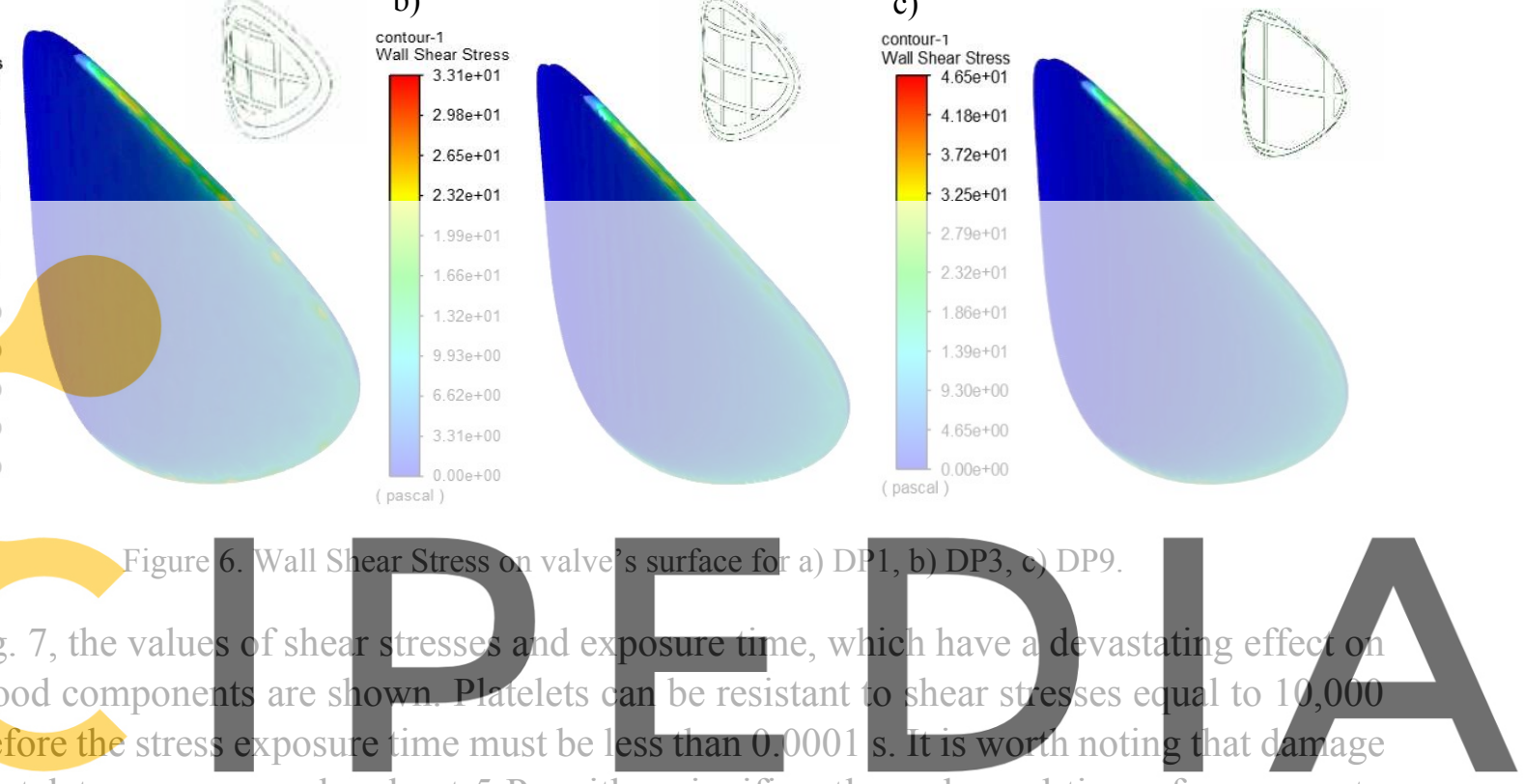

to the platelets may occur already at $5 \mathrm{~Pa}$ with a significantly prolonged time of exposure to

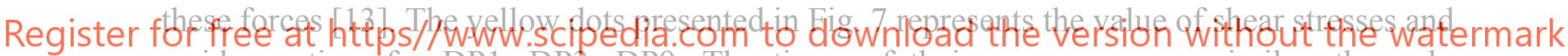
residence time for DP1, DP3, DP9. The times of their exposure were similar, the only differences were observed in the value of stresses. None of the measuring points exceeded the level where platelets could be damaged. Red blood cells show greater resistance to the destructive effect of shear stresses, the exposure time is much extended than for blood platelets [14]. Also, in this case, the maximum shear stresses for the respective measuring points did not exceed the limit in which the hemolysis could occur.

The next steps are connected with some applied statistics methodology, which allows for the development of relationships between parameters that are directly related to the creation of a metamodel, i.e. a formula that takes into account the largest possible area of operation from a limited number of measurement points. Sensitivity analysis is the first point in determining the variables of the greatest importance and thus allows to eliminate unnecessary measurement points, focus on the quantity with the greatest impact on the output. The parameter Imprint has the greatest influence on the value of the maximum shear stresses. Based on the available results, there was no significant effect of other variables. 
a)

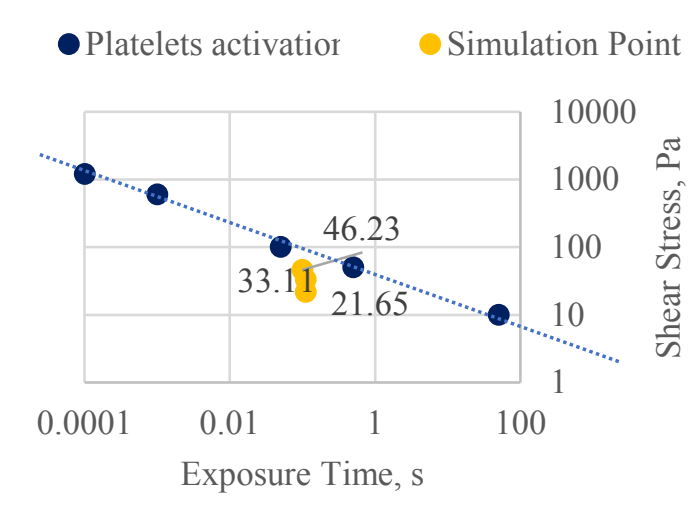

b)

Subhemolytic Damage Point [2 I Simulation point

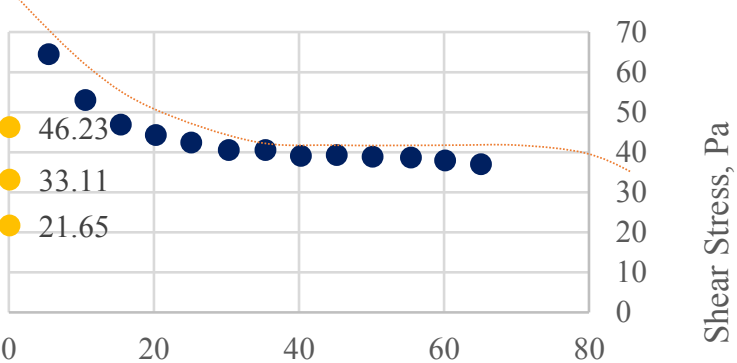

Exposure Time, s

Figure 7. a) The platelets activation value and results and b) subhemolytic damage points and results for DP1, DP3, DP9.
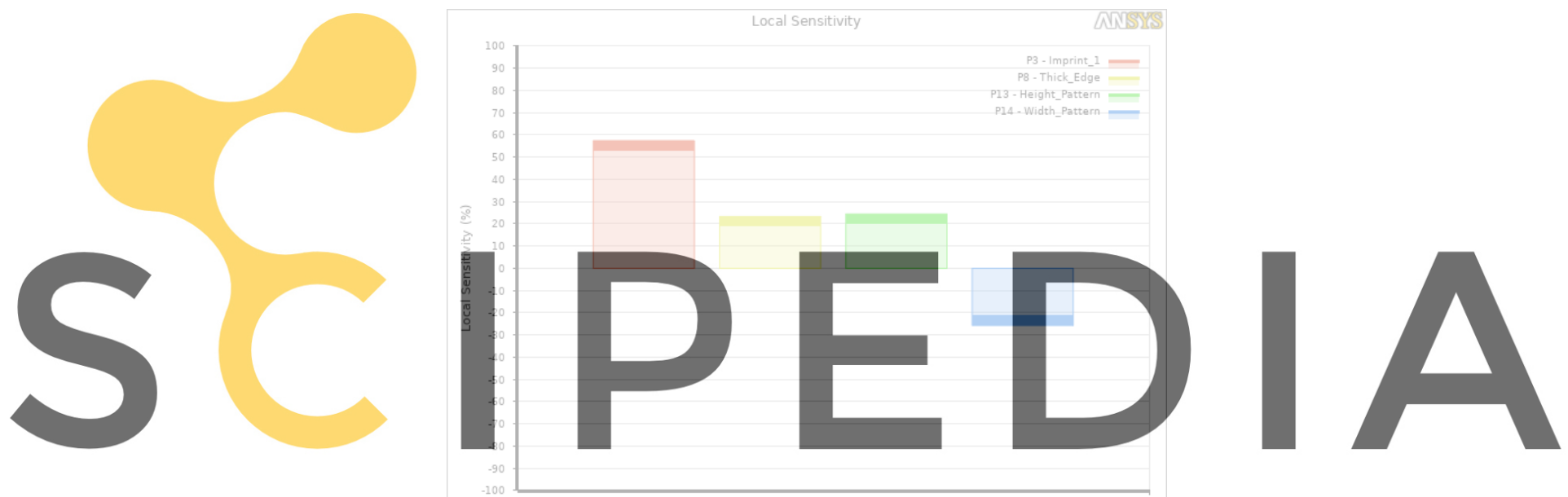

Register for free at https//www.scipedia.com to dow

Figure 8. Local Sensitivity for MaxWallShear output.

The graphical dependencies between explanatory variables and response variables are called response surfaces. Their observation allows verifying the fit of the applied method to the measurement points. The study verified the fitness of three methods: Genetic Aggregation, Neural Network, and Kriging. The relationship between Height_Pattern, Width_Pattern, and MaxWallShear are shown in Figure 9. The shape of the response surface is similar for the Genetic Aggregation and Kriging method, in the Neural Network method the point with the lowest Max Wall Shear value is omitted. Apart from the indicated difference, the general nature of the surface is similar. The smallest MaxWallShear values are on the left side of the chart where Height Patten is the smallest. 
a)

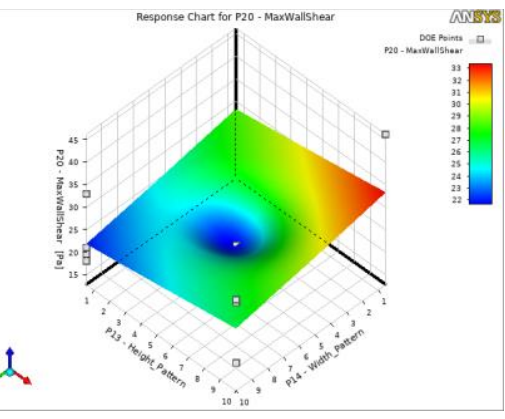

b)

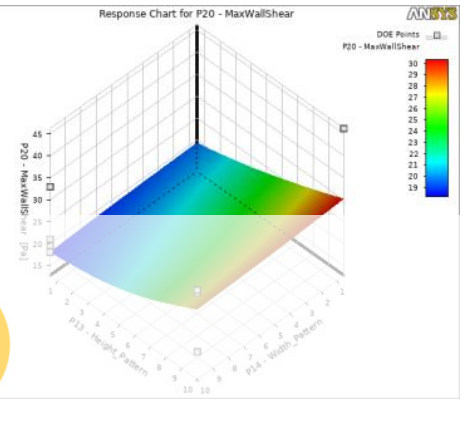

c)

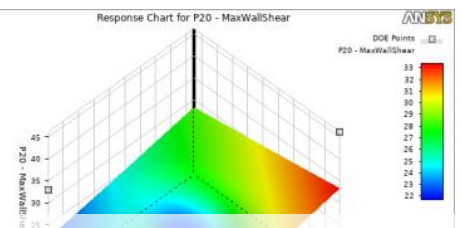

Figure 9. Response Surface for a) Genetic Aggregation, b) Neural Network, c) Kriging method and Height_Pattern, Width_Pattern as input; MaxWallShear as output.
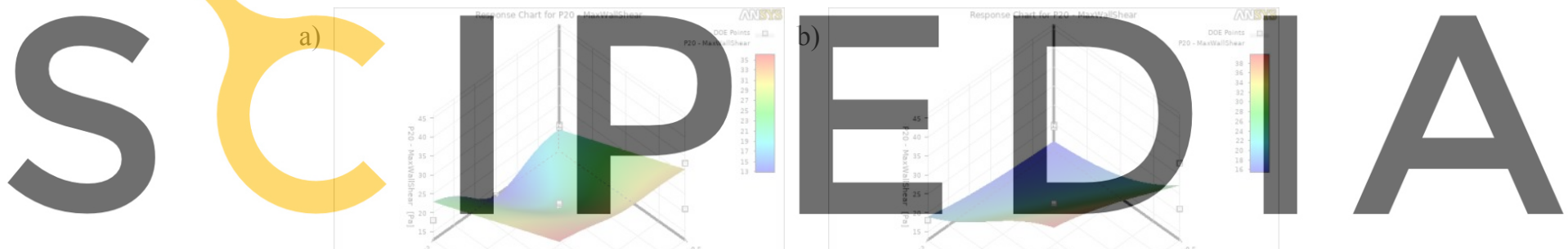

Register for free at https//www.scipedia.com to download the version without the watermark

c)

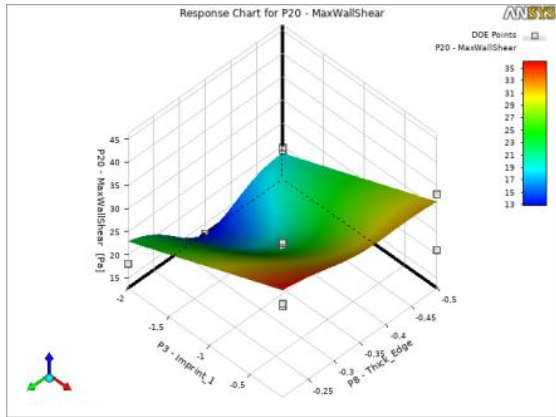

Figure 10. Response Surface for a) Genetic Aggregation, b) Neural Network, c) Kriging method and Imprint 1, Thick Edge as input; MaxWallShear as output.

Similar conclusions can be seen in the case of a response surface for Imprint 1 and Thick_Edge variables (Figure 10). Response surfaces behave similarly for the Genetic 
Aggregation and Kriging methods. Also, the impact of the point with the lowest MaxWallShear value causes the Neural Network's response surface does not to react to changes in the Thick Edge value, as is the case with other considerations, for which the value in the middle of the range provides the lowest possible value of MaxWallShear. The Genetic Aggregation and Kriging methods are the closest to all measuring points when the Neural Network is tightly matched at individual points.

The general optimization problem is defined as in Equation 2. In this study, the objective function was to reduce the maximum value of the shear stress.

$$
\min / \max f_{i}(x) i=1 \ldots N_{o b j}
$$

Subject to:

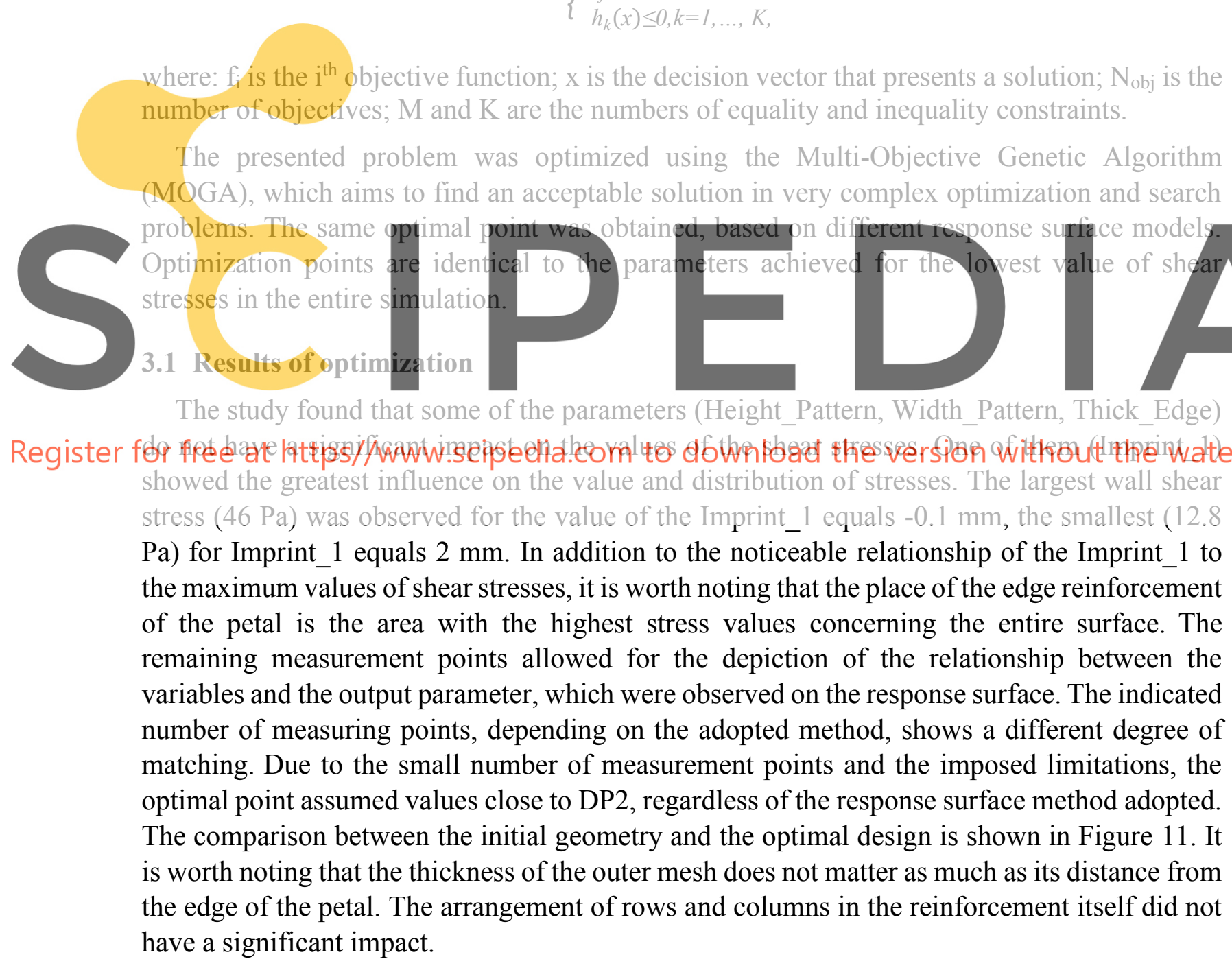

$$
\left\{\begin{array}{l}
g_{j}(x)=0, j=1, \ldots, M \\
h_{k}(x) \leq 0, k=1, \ldots, K
\end{array}\right.
$$

where: $\mathrm{f}_{\mathrm{i}}$ is the $\mathrm{i}^{\text {th }}$ objective function; $\mathrm{x}$ is the decision vector that presents a solution; $\mathrm{N}_{\text {obj }}$ is the number of objectives; $\mathrm{M}$ and $\mathrm{K}$ are the numbers of equality and inequality constraints.

The presented problem was optimized using the Multi-Objective Genetic Algorithm (MOGA), which aims to find an acceptable solution in very complex optimization and search problems. The same optimal point was obtained, based on different response surface models.
Optimization points are identical to the parameters achieved for the lowest value of shear
stresses in the entire simulation.
3.1 Results of optimization

The study found that some of the parameters (Height_Pattern, Width_Pattern, Thick_Edge) showed the greatest influence on the value and distribution of stresses. The largest wall shear stress (46 Pa) was observed for the value of the Imprint_1 equals $-0.1 \mathrm{~mm}$, the smallest (12.8 Pa) for Imprint_1 equals $2 \mathrm{~mm}$. In addition to the noticeable relationship of the Imprint_1 to the maximum values of shear stresses, it is worth noting that the place of the edge reinforcement of the petal is the area with the highest stress values concerning the entire surface. The remaining measurement points allowed for the depiction of the relationship between the variables and the output parameter, which were observed on the response surface. The indicated number of measuring points, depending on the adopted method, shows a different degree of matching. Due to the small number of measurement points and the imposed limitations, the optimal point assumed values close to DP2, regardless of the response surface method adopted. The comparison between the initial geometry and the optimal design is shown in Figure 11. It is worth noting that the thickness of the outer mesh does not matter as much as its distance from have a significant impact. 
a)

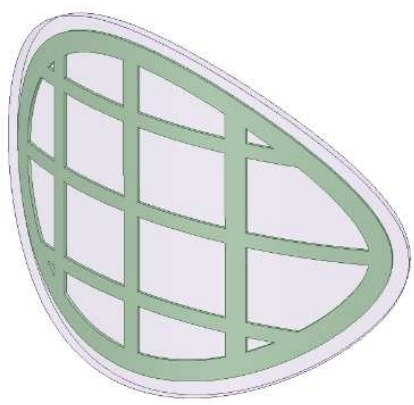

b)

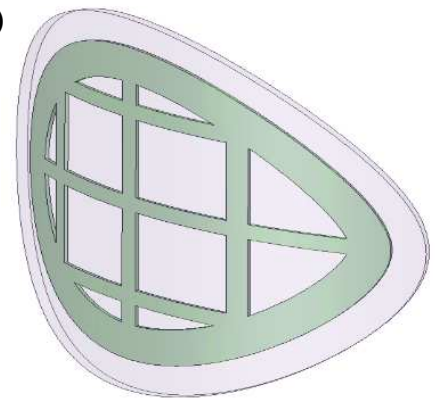

Figure 11. a) Initial and b) optimized valves' design.

The value of the maximum wall shear stress $(12.84 \mathrm{~Pa})$ has been reduced by $40.7 \%$ compared to the initial value $(21.65 \mathrm{~Pa})$. All parameters of the reinforcing mesh are listed in Table 4.

Table 4. Initial and optimized valves parameters.

\begin{tabular}{|c|c|c|c|c|c|c|}
\hline $\mathrm{DP}$ & $\begin{array}{c}\text { Imprint_1, } \\
\mathrm{mm}\end{array}$ & $\begin{array}{c}\text { Thick_Edge, } \\
\mathrm{mm}\end{array}$ & $\begin{array}{c}\text { Height_Pattern, } \\
\mathrm{mm}\end{array}$ & $\begin{array}{c}\text { Width_Pattern, } \\
\mathrm{mm}\end{array}$ & $\begin{array}{c}\text { MaxWallShear, } \\
\mathrm{Pa}\end{array}$ & $\begin{array}{c}\text { MaxWallShear } \\
\text { reduction, \% }\end{array}$ \\
\hline Initial & -1.05 & -0.35 & 5.25 & 5.25 & 21.65 & - \\
\hline Optimized & -2 & -0.35 & 5.25 & 5.25 & 12.84 & 40.7 \\
\hline
\end{tabular}

\section{CONCLUSIONS}

The main aim of the research was to find the main geometric relationships between the mesh reinforcing the valve and the value and the place where the highest wall shear stresses occur. The study used 2-way FSI analysis and various response surface methods. The optimization of the structure was conducted with a Multi-Objective Genetic Algorithm. As a result of the analysis can be found that the sensitivity analysis, is necessary to decide on the parameters that have a significant impact on the nature of the device operation. This is crucial to reduce the insignificant number of parameters and thus the simulation time. The number of measurement points should be increased. Despite the differences in the presentation of meta-models, their operation does not affect the choice of the optimal point but has a significant impact on the imaging of the entire range of device operation. The analysis should cover the entire cardiac cycle, paying attention to the most important issues, the main purpose of the valve operation, preventing backflow. The shear stresses acting on the blood did not contribute to their degradation. The time for which the results are presented is not very representative and should be presented at least for the highest speed in the heart cycle. In future work, the exposure time should be introduced as an additional objective function in the optimization process, where the shear stress value could be reconciled with the corresponding exposure time.

The design of an innovative inlet leaflet valve was used, which can be applicated in the construction of a ventricular assist device and adopted for the needs of other blood-wetted devices. 


\section{References}

[1] G.C. Stewart, M.M. Givertz, Mechanical Circulatory Support for Advanced Heart Failure, Circulation. 125 (2012) 1304-1315.

[2] J. Han, D.R. Trumble, Cardiac assist devices: Early concepts, current technologies, and future innovations, Bioengineering. 6 (2019).

[3] M. Sinnott, P. Cleary, Effect of rotor blade angle and clearance on blood flow through a non-pulsatile, axial, heart pump, Prog. Comput. Fluid Dyn. 10 (2010) 300-306.

[4] Z. Xu, M. Yang, X. Wang, Z. Wang, The influence of different operating conditions on the blood damage of a pulsatile ventricular assist device, ASAIO J. 61 (2015) 656-663.

[5] G. Luraghi, W. Wu, H. De Castilla, J. Félix, R. Matas, G. Dubini, P. Dubuis, M. Grimmé, F. Migliavacca, Numerical Approach to Study the Behavior of an Artificial Ventricle : Fluid - Structure Interaction Followed by Fluid Dynamics With Moving Boundaries, 42 (2018).

[6] K.H. Fraser, M.E. Taskin, B.P. Griffith, Z.J. Wu, The use of computational fluid dynamics in the development of ventricular assist devices, Med. Eng. Phys. 33 (2011) 263-280.

[7] B. Ghadimi, A. Nejat, S.A. Nourbakhsh, N. Naderi, Multi-Objective Genetic Algorithm Assisted by an Artificial Neural Network Metamodel for Shape Optimization of a Centrifugal Blood Pump, (2018).

[8] C.C.L.A.L.M.Y. Bazilevs, Shape optimization of pulsatile ventricular assist devices using FSI to minimize thrombotic risk Shape optimization of pulsatile ventricular assist devices using FSI to minimize thrombotic risk, (2013).

[9] Y. Cho, Effects of the non-Newtonian viscosity of blood on flows in a diseased arterial vessel . Part 1 : Steady flows, (2014).

[10] M. Kopernik, P. Tokarczyk, Development of multi-phase models of blood flow for medium-sized vessels with stenosis, Acta Bioeng. Biomech. 21 (2019).

[11] T. Kenner, The measurement of blood density and its meaning, Basic Res. Cardiol. 84 (1989) 111-124.

[12] R.A. Malinauskas, P. Hariharan, S.W. Day, L.H. Herbertson, M. Buesen, U. Steinseifer, K.I. Aycock, B.C. Good, S. Deutsch, K.B. Manning, B.A. Craven, FDA Benchmark Medical Device Flow Models for CFD Validation., ASAIO J. 63 (2017) 150-160.

[13] K.H. Fraser, T. Zhang, M.E. Taskin, B.P. Griffith, Z.J. Wu, A quantitative comparison of mechanical blood damage parameters in rotary ventricular assist devices: shear stress, exposure time and hemolysis index, J. Biomech. Eng. 134 (2012) 81002.

[14] M. Simmonds, H. Meiselman, Prediction of the level and duration of shear stress exposure that induces subhemolytic damage to erythrocytes, Biorheology. 53 (2017) 113. 